\title{
Developments towards the delivery of selenium ion beams at ISOLDE
}

\author{
K. Chrysalidis ${ }^{1,2, a}$, J. Ballof ${ }^{1,3, b}$, Ch.E. Düllmann ${ }^{3,4,5}$, V.N. Fedosseev ${ }^{1}$, C. Granados ${ }^{1}$, B.A. Marsh ${ }^{1}$, \\ Y. Martinez Palenzuela ${ }^{1,6}$, J.P. Ramos ${ }^{1}$, S. Rothe ${ }^{1}$, T. Stora ${ }^{1}$, and K. Wendt ${ }^{2}$ \\ 1 CERN, 1211 Geneva, Switzerland \\ 2 Institut für Physik, Johannes Gutenberg-Universität, 55099 Mainz, Germany \\ 3 Institiut für Kernchemie, Johannes Gutenberg-Universität, 55099 Mainz, Germany \\ 4 Helmholtz-Institut Mainz, 55099 Mainz, Germany \\ 5 GSI Helmholtzzentrum für Schwerionenforschung, 64291, Darmstadt, Germany \\ ${ }^{6}$ KU Leuven, Instituut voor Kern- en Stralingsfysica, 3001 Leuven, Belgium
}

Received: 24 April 2019 / Revised: 15 August 2019

Published online: 14 October 2019

(C) CERN 2019. This article is published with open access at Springerlink.com

Communicated by K. Blaum

\begin{abstract}
The production of selenium ion beams has been investigated at the CERN-ISOLDE facility via two different ionization methods. Whilst molecular selenium (SeCO) beams were produced at ISOLDE since the early 1990s, recent attempts at reliably reproducing these results have so far been unsuccessful. Here we report on tests of a step-wise resonance laser ionization scheme for atomic selenium using the ISOLDE Resonance Ionization Laser Ion Source (RILIS). For stable selenium an ionization efficiency of $1 \%$ was achieved. During the first on-line radioisotope production tests, a yield of $\approx 2.4 \times 10^{4}$ ions $/ \mu \mathrm{C}$ was measured for ${ }^{71} \mathrm{Se}^{+}$, using a $\mathrm{ZrO}_{2}$ target with an electron impact ion source. In parallel, an approach for extraction of molecular carbonyl selenide ( $\mathrm{SeCO})$ beams was tested. The same ion source and target material were used and a maximum yield of $\approx 3.6 \times 10^{5} \mathrm{ions} / \mu \mathrm{C}$ of ${ }^{71} \mathrm{SeCO}^{+}$was measured.
\end{abstract}

\section{Introduction}

CERN-ISOLDE is an isotope separation on-line facility [1, 2 ], where isotopes can be produced, then ionized and extracted within one apparatus, often in a selective (isotope purity) and efficient (up to several percent overall efficiency) process. This method provides the means to supply ion beams of exotic isotopes, far from stability, with half-lives as low as several ms [3]. For this purpose, protons, provided by the CERN Proton Synchrotron Booster (PSB) with an energy of $1.4 \mathrm{GeV}$, impinge on a thick target. Reaction products created by spallation, fragmentation or fission are then evaporated from the target material and ionized. The target and ion source assembly is situated on a $20-60 \mathrm{kV}$ high voltage platform and is resistively heated causing the reaction products to diffuse through the target material and effuse into the ion source. Once the atoms or molecules have been ionized, they are extracted by the grounded extraction electrode situated downstream of the ion source. The temperature of ion

\footnotetext{
${ }^{a}$ e-mail: katerina.chrysalidis@cern.ch (corresponding author)

b e-mail: jochen.ballof@cern.ch
}

source and target container are controlled by two separate electrical circuits, allowing to independently adjust the temperatures within a wide range.

Three main methods are used at ISOLDE to produce ion beams: thermal ionization on a hot metal surface, electron impact ionization in a FEBIAD-type (Forced Electron Beam Induced Arc Discharge) ion source or VADIS (Versatile Arc Discharge Ion Source) [4], and laser resonance ionization. The laser-atom interaction can take place in different environments: a hot cavity surface ion source [5], a radio-frequency ion guide LIST (Laser Ion Source and Trap) [6] or a VADIS operated at reduced anode voltage $[7,8]$.

Several attempts have been made to produce selenium beams at ISOLDE in the past, namely for Coulomb excitation studies using post-accelerated ${ }^{70}$ Se [9]. Such an experiment requires a beam purity in the order of $90 \%$. Due to the abundance of isobaric contaminants expected at the desired atomic masses, un-selective FEBIAD ionization of elemental selenium is not expected to yield the required purity for the proposed Coulomb-excitation experiment using HIE-ISOLDE [10,11]. Even the element selective RILIS process might not yield the required purity, since selenium $(Z=34)$ is located half-way between the 
surface ionizable elements gallium $(Z=31)$ and rubidium $(Z=37)$. Isobars of one of these elements tend to be produced at comparable or higher yields for most Se isotopes. Thus all neutron-rich Se isotopes $(A>80)$ produced from $\mathrm{GeV}$ proton-induced fission of any type of $\mathrm{U}$ or $\mathrm{Th}$ target will likely suffer from isobaric $\mathrm{Rb}$, while spallationproduced neutron-deficient Se isotopes up to about ${ }^{73} \mathrm{Se}$ will suffer from isobaric Ga. In some cases the isobars are stable $\left({ }^{67,69} \mathrm{Ga}\right.$ and $\left.{ }^{85,87} \mathrm{Rb}\right)$, allowing decay spectroscopy studies. However, these isobars will still affect any type of experiment that is disturbed by stable contaminants, due to ion load limitations (such as ion beam bunching and charge breeding before post-acceleration). However, for the molecular beam ${ }^{x} \mathrm{SeCO}^{+}$, fewer contaminant isobars are expected, which are mainly ${ }^{27} \mathrm{Al}^{x+1} \mathrm{Br}^{+}[12]$ and ${ }^{x-4} \mathrm{Ge}^{32} \mathrm{~S}^{+}$.

In a past experiment, contamination from isobaric ${ }^{72} \mathrm{Ga}^{+}$was avoided by extracting selenium in the form of a $\mathrm{SeCO}^{+}$beam, through the use of a FEBIAD-type ion source. The $\mathrm{SeCO}^{+}$was subsequently broken up inside the Electron Beam Ion Source (EBIS) of the REX-ISOLDE and the resulting $\mathrm{Se}^{n++}$-ions were post-accelerated. The disadvantage of this approach is that a significant amount of the ions of interest are lost, due to the distribution among different molecular fractions [13]. Furthermore, many low-energy experiments at ISOLDE, which are situated upstream of the EBIS, are not readily able to make use of molecular beams (solid state physics implantations, collinear laser spectroscopy, etc.). These experiments would profit from the production of elemental $\mathrm{Se}^{+}$beams. The Resonance Ionization Laser Ion Source (RILIS) [14], which is the most commonly used ion source at ISOLDE, can provide this alternative and generate an element-selective way of ionizing selenium.

\section{Laser ionization of selenium atoms}

\subsection{Atomic structre of selenium}

Selenium belongs to the chalcogens (oxygen group) along with oxygen $(\mathrm{O})$, sulfur $(\mathrm{S})$, tellurium $(\mathrm{Te})$ and polonium $(\mathrm{Po})$. Chalcogens have six valence electrons and relatively high ionization energies, which decrease with increasing atomic number. They are therefore not efficiently surface ionized in the typical $\mathrm{Ta}, \mathrm{W}$ or Re surface ion source at maximum temperatures of around $1800-2500^{\circ} \mathrm{C}$. At ISOLDE, laser ionization schemes for Te and Po have been developed and applied in past experiments $[15,16]$.

The information on the atomic level structure of Se atom available in [17] is mostly based on the spectral data obtained in $[18,19]$. The electronic configuration of the Se atomic ground state is $[\mathrm{Ar}] 4 s^{2} 4 p^{4}{ }^{3} \mathrm{P}$ with $J=2$. The state $[\mathrm{Ar}] 4 s^{2} 4 p^{4}{ }^{3} \mathrm{P}$ forms a triplet as shown in fig. 1. An estimation of thermal population of these states can be derived from Boltzmann distribution calculations. At $T=2000^{\circ} \mathrm{C}$ (used for the measurements, see sect. 4), 83\% of the ${ }^{3} \mathrm{P}_{2}$ state is populated, whilst the ${ }^{3} \mathrm{P}_{1}$ and ${ }^{3} \mathrm{P}_{0}$ states are populated with $14 \%$ and $3 \%$ respectively. The lowest-lying energy level accessible via a single-photon transition is the

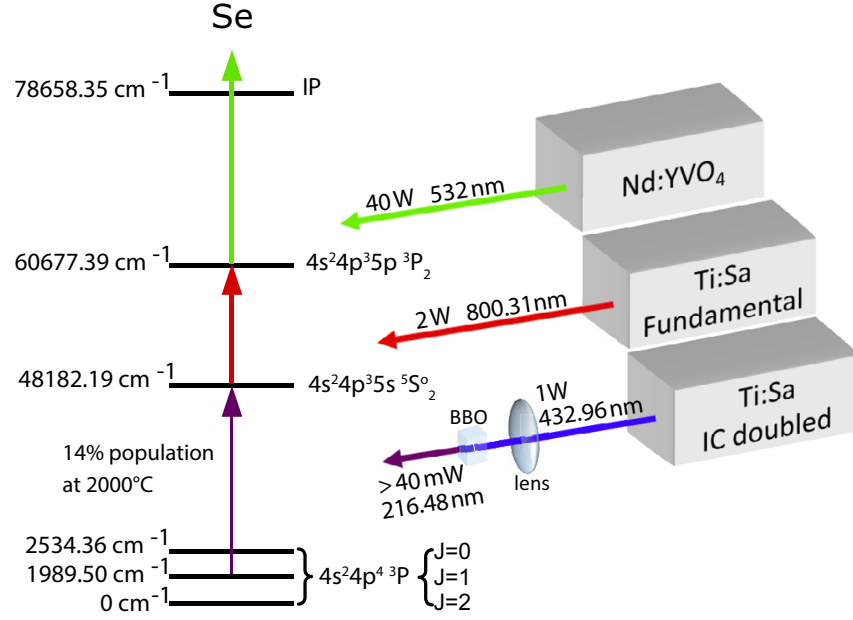

Fig. 1. Ionization scheme for atomic selenium alongside the RILIS lasers which provided the excitation steps with their respective powers. The first step is provided by intra-cavity (IC) frequency doubling a Ti:Sapphire laser and subsequent frequency doubling of this blue output in a single pass through a BBO crystal to generate UV light at $216.5 \mathrm{~nm}$. The Ti:Sapphire lasers are pumped by frequency doubled $\mathrm{Nd}: \mathrm{YAG}$ lasers at $532 \mathrm{~nm}$. Typical pulse lengths are $\approx 50 \mathrm{~ns}$. The $\mathrm{Nd}_{\mathrm{YVO}}$ is a BLAZE laser from Coherent. The population for the metastable level used for the chosen first step excitation is given. Level configurations and energies are from [17], vacuum wavelengths are given.

$4 s^{2} 4 p^{3} 5 s^{5} \mathrm{~S}_{2}^{o}$ state at an energy of $48182.19 \mathrm{~cm}^{-1}$. Excitation to this state can be achieved either from the ground state ${ }^{3} \mathrm{P}_{2}\left(207.55 \mathrm{~nm}_{\mathrm{vac}}\right)$ or from the first metastable state ${ }^{3} \mathrm{P}_{1}\left(216.48 \mathrm{~nm}_{\mathrm{vac}}\right)$. The transition from the ground state is the preferred option for optimal ionization efficiency due to the thermal population distribution.

\subsection{RILIS laser set up and ionization scheme development}

The current status of the ISOLDE RILIS is described in detail in reference [14]. Typically 2-3 tunable, pulsed $(10 \mathrm{kHz}$ repetition rate) laser beams are temporally and spatially overlapped inside the ion source cavity and tuned to an element-specific multi-step resonance ionization scheme. The optimal ionization scheme is typically determined for each element by a dedicated resonance ionization spectroscopy study. An ideal ionization scheme is one that combines maximum efficiency with a convenient (reliable, easy to set up and maintain) laser configuration.

For generating the first excitation step, an attempt was made to produce $207.55 \mathrm{~nm}_{\mathrm{vac}}$ light by 4 th harmonic generation using a Ti:sapphire laser with an intra-cavity second harmonic generation (SHG) and focusing its $\approx 1 \mathrm{~W}$ blue output with a $150 \mathrm{~mm}$ focal length lens, into a $6 \times 5 \times 4 \mathrm{~mm}$ BBO crystal (type $1 \mathrm{SHG}$ - angle $79.7^{\circ}$, supplied by CRYSTECH INC). Although up to $50 \mathrm{~mW}$ at $207.55 \mathrm{~nm}_{\text {vac }}$ was achieved, destructive absorption of 
Table 1. Second step transitions investigated during the ionization scheme development for selenium, with the first step set to $216.48 \mathrm{~nm}_{\mathrm{vac}}$. The relative ion beam intensities achieved using these second steps, compared to the most efficient ionization scheme using the $4 p^{3} 5 p^{3} \mathrm{P}_{2}$ state are given. The expected laser power transmission to the ion source is $\approx 70 \%$ for the non-resonant step at $532 \mathrm{~nm}$. The 5 new transitions that were found are marked in bold.

\begin{tabular}{c|c|c|c} 
2nd step $\Delta E\left[\mathrm{~cm}^{-1}\right]$ & $E_{\text {total }}\left[\mathrm{cm}^{-1}\right]$ & 3rd step & rel. int. \\
\hline 12495.20 & 60677.39 & $532 \mathrm{~nm}$ & 1 \\
\hline 24901.15 & 73082.34 & scan for AI & \\
\hline 24912.21 & 73094.40 & & $5 \times 10^{-3}$ \\
24870.10 & 73052.29 & $532 \mathrm{~nm}$ & $7 \times 10^{-3}$ \\
24860.73 & 73042.92 & & $3 \times 10^{-2}$ \\
24435.99 & 72618.18 & & $1 \times 10^{-5}$ \\
\hline $\mathbf{1 7 3 7 6 . 5 ( 2 )}$ & $\mathbf{6 5 5 5 8 . 7 ( 2 )}$ & & \\
$\mathbf{1 7 3 8 0 . 4 ( 2 )}$ & $\mathbf{6 5 5 6 2 . 6 ( 2 )}$ & & \\
$\mathbf{1 7 4 2 2 . 0 ( 2 )}$ & $\mathbf{6 5 6 0 4 . 2 ( 2 )}$ & $532 \mathrm{~nm}$ & $1 \times 10^{-5}$ \\
$\mathbf{1 7 7 4 4 . 5 ( 2 )}$ & $\mathbf{6 5 9 2 6 . 7 ( 2 )}$ & & \\
$\mathbf{1 7 7 9 5 . 5 ( 2 )}$ & $\mathbf{6 5 9 7 7 . 7 ( 2 )}$ & &
\end{tabular}

this wavelength in the BBO crystal occurred within minutes. The severe loss of 4 th harmonic generation efficiency therefore renders this approach impractical.

Therefore, the metastable state with the estimated population of $14 \%$ was used for the measurements described in this work (see fig. 1). The clear disadvantage is an expected loss in efficiency of a factor $\approx 6$, due to the lower thermal population at $2000{ }^{\circ} \mathrm{C}$ compared to the ground state. The required wavelength of $216.48 \mathrm{~nm}_{\mathrm{vac}}$ was generated by 4 th harmonic generation of a Ti:sapphire laser operating at $865.92 \mathrm{~nm}_{\mathrm{vac}}$. The same method described above, this time with a BBO crystal at $69.7^{\circ}$, was used.

For the initial verification of the laser beam position and overlap, transitions known from the literature [18] were tested as second steps. An additional laser, operating at $532 \mathrm{~nm}$ with a power of $40 \mathrm{~W}(10 \mathrm{kHz}$ repetition rate and 17 ns pulse length), was used for non-resonant ionization into the continuum. The power of this laser was stable for all measurements listed in table 1 and the position inside the ion source remained fixed through the use of an active beam stabilization system. The first of the second-step transition tested leads to the $4 s^{2} 4 p^{3} 5 p^{3} \mathrm{P}_{2}$ level at a total energy of $60677.388 \mathrm{~cm}^{-1}$, corresponding to a required transition wavelength of $\approx 800.31 \mathrm{~nm}_{\mathrm{vac}}$. The alternative transition to the $4 s^{2} 4 p^{3} 5 p^{3} \mathrm{P}_{1}$ state was not tested due to the lower $J$ quantum number of this state. The lower occupancy of this level $(2 J+1)$ is expected to result in a reduced maximum ionization efficiency [20]. Several other transitions that can be found in literature were tested and are listed in table 1 . The relative intensities compared to the ionization pathway via the $4 s^{2} 4 p^{3} 5 p^{3} \mathrm{P}_{2}$ level were of the order of $1 \times 10^{-5}-3 \times 10^{-2}$. No further investigations into these states were performed since, based on previous experience, it is not reasonable to expect that a transition to an autoionizing state from these levels would yield more than a factor 3-5 enhancement in ionization efficiency [21].

Therefore, a search for new second-step transitions was performed by scanning the second step laser wavelength in the range of $720-920 \mathrm{~nm}$ and $558-592 \mathrm{~nm}$. These scans were carried out using respectively a grating-based Ti:sapphire laser and a Rhodamine 6G dye laser available at the RILIS laser laboratory. In the range covered by the Ti:sapphire laser, no second-step transitions were found. The wavelength scan of the dye revealed five resonances, which could not be attributed as transitions from the $4 s^{2} 4 p^{3} 5 s{ }^{5} \mathrm{~S}_{2}^{o}$ state to any of the higher levels tabulated in [17]. Since each of those resonances was observable only under presence of the laser beam at $216.48 \mathrm{~nm}_{\mathrm{vac}}$, it is reasonable to conclude that new excited levels, linked by the optical transitions with the $4 s^{2} 4 p^{3} 5 s{ }^{5} \mathrm{~S}_{2}^{o}$ state, were found. The transitions and respective levels are listed in table 1 . It is worth to mention that the table of lines observed in the arc spectrum of selenium (see [18]) contains four lines with frequencies very close to the newly found transitions $\left(17376.46 \mathrm{~cm}^{-1}\right.$, $17380.37 \mathrm{~cm}^{-1}, 17421.99 \mathrm{~cm}^{-1}$ and $\left.17795.41 \mathrm{~cm}^{-1}\right)$. However, the authors of [18] did not designate these transitions to the level used in our work. Neither of the transitions resulted in a sufficiently intense ion rate to be considered as a part of a favorable ionization scheme.

\subsection{Laser ionization efficiency measurement}

The RILIS efficiency is measured as the percentage of a calibrated-mass sample that is extracted and detected as an ion beam during optimal RILIS conditions. A sample with a calibrated amount of selenium (natural abundance) atoms (in standard solution of $5 \% \mathrm{HNO}_{3}$ on Ta foil) was inserted into one of the resistively heated capillary ovens, referred to as "mass markers" [22]. This was attached to the transfer line of the empty target container, as is schematically depicted in fig. 2 . The sample was gradually evaporated over $>5$ hours and the ion current was measured using a Faraday cup during this time. Integrating this measurement over the evaporation time reveals the total number of ions that reach the detection point (after extraction and mass separation).

The efficiency was determined to be $\approx 0.6 \%$. Due to instabilities in the UV generation for the first step excitation, the power of this step was deliberately limited to $40 \mathrm{~mW}$ by moving the 4 th harmonic generation crystal away from the focus of the intra-cavity generated blue light. Absorption-induced damage to the crystal is then reduced, resulting in a more stable operation. When reducing the power of the first step from $\approx 80 \mathrm{~mW}$ (on the laser table) to $40 \mathrm{~mW}$, a drop in ion current of factor 2 was observed. If the UV light is kept at $80 \mathrm{~mW}$, a correction with a factor of 2 can be applied to the calculated result from the data. Previously the RILIS at ISOLDE (or TRIUMF respectively) has been operated over extensive periods with UV light of comparable wavelengths, e.g. for ionization of $\mathrm{Zn}(65 \mathrm{~mW}$ on the table at $213.9 \mathrm{~nm})$ or $\mathrm{Sb}(40 \mathrm{~mW}$ on the table at $217.6 \mathrm{~nm})$ respectively (see 


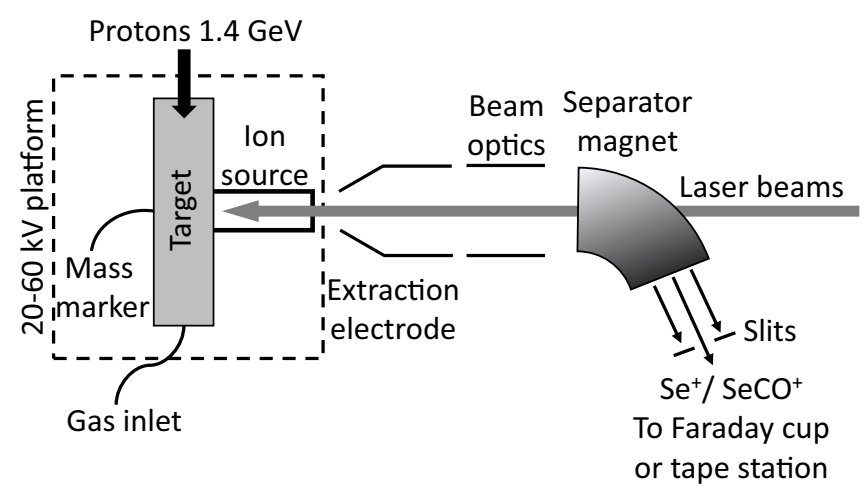

Fig. 2. Schematic view of the experimental set up (not to scale) of the target and ion source. Laser beams were directed into the ion source through a window in the mass separator vacuum chamber. The gas inlet for the production of molecular Se beams was attached to the side of the target container and the mass marker to the back of the transfer line. More details are given in the text.

also [23], table 4). Thus, stable operation with at least $65 \mathrm{~mW}$ of UV light can be guaranteed, leading to the efficiency value of $1 \%$.

No uncertainty is quoted for the efficiency measurement since only a single measurement was performed. Nevertheless, this type of ISOL efficiency measurement is known to be susceptible to fluctuations due to several contributing factors such as an accumulation of the sample in cold spots of the target/ion source assembly, losses of a portion of the sample in preparation and initial target heating. The value should therefore be considered as a lower limit. Should reliable production of $207.5 \mathrm{~nm}_{\mathrm{vac}}$ for a first step transition from the ground state become feasible, a laser ionization efficiency of about $10 \%$ can be expected, due to the higher thermal population of this state.

\section{Production of molecular selenium}

Molecular selenium beams were first reported in 1992 by Hagebø et al. [24] who operated a $\mathrm{ZrO}_{2}$ fiber target equipped with a FEBIAD source under an additional oxygen partial pressure of $7 \times 10^{-4}$ mbar. The selenium was extracted as carbonyl selenide ( $\mathrm{SeCO}$ ) and allowed spectroscopic studies on the isotopes ${ }^{67,68} \mathrm{Se}$. However, the exact mechanism of molecular formation remained unclear. Parts of the ion source assembly were proposed as source of carbon which was likely emitted by the graphite grid of a FEBIAD MK5 ion source [25]. Also the source of oxygen remained unclear since the absence of SeCO beams was not confirmed prior to oxygen injection, and after discontinuing the oxygen injection, the SeCO beams did not deplete. A partial pressure of free oxygen is expected from the hot oxide target material itself. Despite this intrinsic presence of oxygen, the influence of additional oxygen supply was tested in 2016 at ISOLDE. Air, containing ca. $21 \%$ oxygen, was injected through a calibrated leak of $3.7 \times 10^{-5}$ mbar L s $^{-1}$ into a zirconia fiber target, as shown schematically in fig. 2. After injection, the radioactive ion yields of ${ }^{67} \mathrm{Ge}^{32} \mathrm{~S}^{+}$and ${ }^{71} \mathrm{SeCO}^{+}$decreased by factors of ten and two, respectively. A more recent suggestion is that SeCO formation is fostered by injection of carbon dioxide gas, which decomposes to carbon monoxide and oxygen at typical operation temperatures of the target and ion source. This in turn, would open up the classic synthetic route for carbonyl selenide (SeCO) [26].

The production mechanism of the compound inside the target unit was investigated, since issues regarding the stability of ion beam extraction had been observed. A steep decrease in the yields of carbonyl selenide was found, the most likely cause being a depletion of the carbon or oxygen source. To investigate the formation mechanism, tests at the ISOLDE off-line mass separator [27] were performed using target unit \#605 which was equipped with a VADIS ion source [4], $\mathrm{ZrO}_{2}$ fibers inside the tantalum target container and an oven supplying selenium. The setup allowed to study the influence of operation parameters on SeCO formation, which could be determined as the ratio between the extracted beams of nat $\mathrm{Se}^{+}$and ${ }^{n a t} \mathrm{SeCO}^{+}$. Target container and ion source cathode temperatures have been deduced from a calibration curve obtained by variation of the applied heating current, while monitoring the temperature with a pyrometer. The temperature of the ion source was measured at the cathode of the ion source assembly. Whilst varying the ion source cathode temperature in the range from 1960 to $2100^{\circ} \mathrm{C}$ did not significantly affect the SeCO formation, a substantial influence of the target container temperature on the SeCO formation was observed. At low temperatures, the extraction is shifted towards the atomic selenium. The target unit was additionally equipped with a calibrated leak to introduce $99 \%$ isotopically enriched ${ }^{13} \mathrm{CO}_{2}$ into the ion source. The rate of the leak was initially chosen to be $2.5 \times 10^{-5} \mathrm{mbar} \mathrm{L} \mathrm{s}^{-1}$ and was later increased to $1.0 \times 10^{-4} \mathrm{mbar} \mathrm{L} \mathrm{s}^{-1}$ and $1.2 \times 10^{-3} \mathrm{mbar} \mathrm{L} \mathrm{s}^{-1}$. The corresponding ${ }^{13} \mathrm{CO}$ partial pressures of $4.0 \times 10^{-5}, 1.6 \times 10^{-4}$ and $1.9 \times 10^{-3}$ mbar, respectively, are expected to form in the ion source, based on the conductance estimation in the molecular flow regime and assuming, that $\mathrm{CO}_{2}$ quantitatively breaks up to $\mathrm{CO}$, in agreement with the Boudouard equilibrium.

The presence of ${ }^{13} \mathrm{C}$ inside the ion source could be verified in the extracted beams. For the largest leak rate, $177 \mathrm{nA}$ of ${ }^{12} \mathrm{CO}^{+}$and $2330 \mathrm{nA}$ of ${ }^{13} \mathrm{CO}^{+}$were measured, while the current on the mass of ${ }^{13} \mathrm{CO}^{+}$was below $3 \mathrm{nA}$ before injection of ${ }^{13} \mathrm{CO}_{2}$. Despite the expectations, the extracted nat $\mathrm{SeCO}$ beams are dominated by the ${ }^{12} \mathrm{C}$ isotope, and ${ }^{\text {nat }} \mathrm{Se}^{13} \mathrm{CO}$ could not be unambiguously identified in the mass spectrum. Thus, the results indicate that the injected carbon dioxide does not serve as primary carbon source for carbonyl selenide formation. Furthermore, the formation of SeCO is significantly affected by the target temperature, as discussed in more detail in sect. 4 .

An elementary analysis by electron-dispersive X-ray spectroscopy (EDS) did not show carbon above the detection limit of typically $0.1 \%$ in the zirconia target material itself. While the result shows, that no important carbon fraction is present in the material, a contribution of carbon e.g. originating from the decomposition of minor 
amounts of zirconium carbide at elevated temperatures cannot be excluded. Another source of carbon are the structural parts of the target assembly, which is obvious from intense $\mathrm{CO}^{+}$beam typically extracted from freshly assembled target units without charge in the target container.

So far, injection of tetrafluoromethane $\left(\mathrm{CF}_{4}\right)$ was found to regenerate radioactive SeCO beams after depletion of the sideband, albeit at a lower yield. Further investigations are ongoing.

\section{On-line yield measurements with $\mathrm{ZrO}_{2}$ as target material}

Two different target units (\#605 and \#612) with Yttriastabilized zirconia fibers as target material were used for carbonyl selenide ion beam production. The target material was supplied by Zircar Zirconia Inc., PO BOX 287 87 Meadow Road, NY 10921 Florida, type ZYBF-5, lot \#30D-96. Both target units were equipped with FEBIADtype ion sources. Due to the decreasing production of SeCO during operation of the first target unit \#605, it was investigated if the carbon inventory of the original FEBIAD MK5 source [25] can be exploited to foster SeCO formation. The earlier FEBIAD sources contained graphite parts (accelerating grid, outer ring of outlet plate), which were found responsible for a substantial carbon monoxide beam at elevated temperatures. These parts have been replaced by molybdenum within the development of the VADIS ion source [27]. Therefore for target unit \#612 the molybdenum grid of the VADIS source was replaced by a graphite grid. Other than this part, the ion sources were identical. To minimize the reduction of the carbon inventory during offline conditioning, the heating time of the target before online operation was minimized for unit \#612. This resulted in an initial $\mathrm{CO}^{+}$ current of $540 \mathrm{nA}$ for target unit \#612, which was ca. five times the value of target unit \#605, measured under comparable conditions.

Due to this additional supply of carbon, an enhancement of molecular formation of SeCO was therefore expected for the second target, unfortunately, this was not the case. Additionally, a comparison between a VADISionized molecular beam of $\mathrm{SeCO}^{+}$and a resonantly laser ionized atomic beam in VADLIS mode (running with an anode voltage $<10 \mathrm{~V}$ ) [7] was made with target \#605. For the VADLIS measurements laser powers were optimized for efficiency, meaning that the first step was operated at $80 \mathrm{~mW}$.

Yields of radioactive species for both target units were measured using gamma-ray spectroscopy with the ISOLDE tapestation [28], located at the central beamline, which is equipped with a plastic scintillator and a high purity germanium detector. The obtained yields strongly depend on the history of target heating and temperatures during the measurement. The yields for the different ionization mechanisms are given in fig. 3, alongside the temperature dependence and time evolution in fig. 4 . Due to

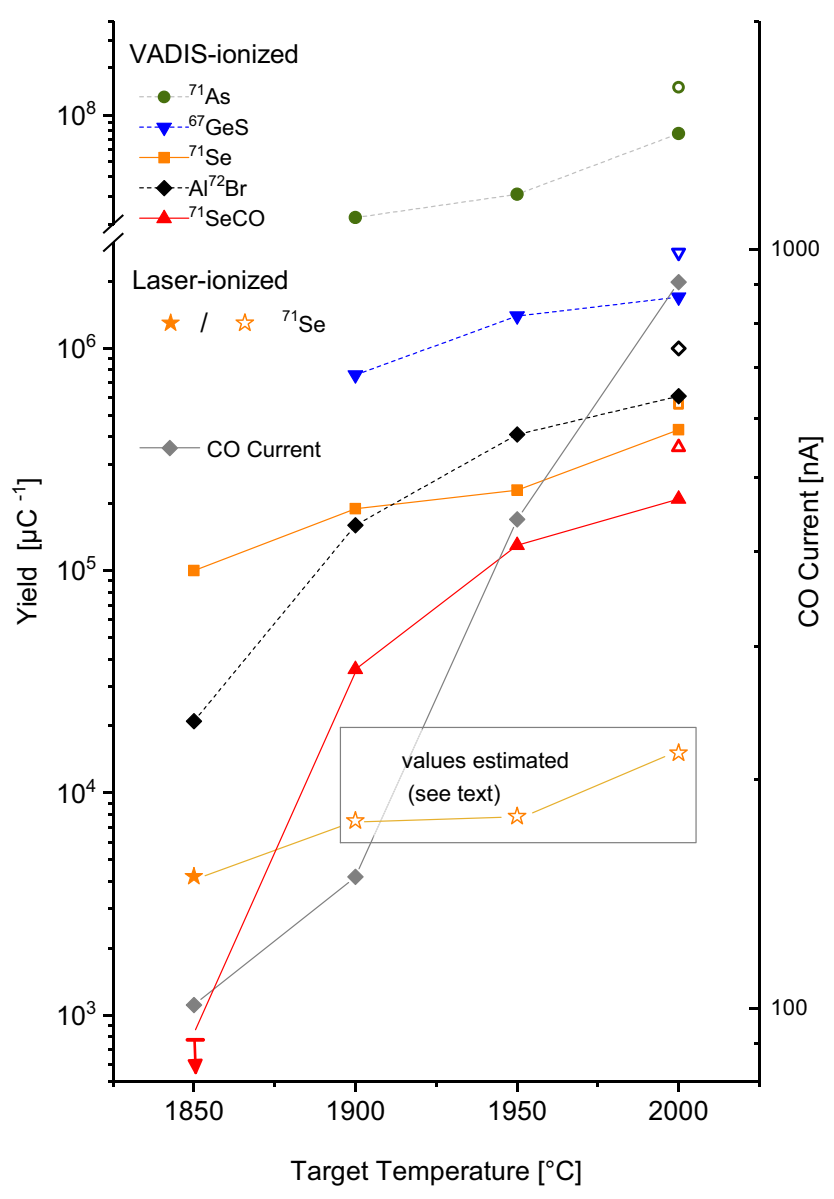

Fig. 3. Radioactive ion beam yields obtained from target \#605 $[\mathrm{ZrO}]$ at the beginning of the run in dependence of the target container temperature. Open symbols represent measurements at an ion source cathode temperature of $2100{ }^{\circ} \mathrm{C}$, otherwise the ion source cathode temperature was $2050^{\circ} \mathrm{C}$. The yield of laser-ionized ${ }^{71} \mathrm{Se}^{+}$was only measured at a target temperature of $1850{ }^{\circ} \mathrm{C}$, laser-ionized yields at higher temperatures were estimated by eq. (1). See text for details.

limited availability of beam time, the yield of laser ionized selenium could only be measured at the target temperature of $1850{ }^{\circ} \mathrm{C}$. Contrarily, significant yields of molecular species were only present at higher target temperatures. It is well established that the fraction of radioactive species released from the target material before their decay typically increases with target temperature (see e.g. [29]). For prospective users of laser-ionized selenium beams, it is of interest to estimate the yield at higher temperatures. Thus, an estimation of expected laser-ionized selenium yields is required, which also allows a comparison of electron-impact and laser ionization yields. Within the given order of intensity $(<1 \mathrm{pA})$, the radioactive beam intensity scales linearly with the amount of neutrals introduced into the ion source. The latter equally holds for laser and electron impact ionization. Measurements with the FEBIAD source allow an estimation of the increase in atomic selenium injected into the ion source. In the FEBIAD source two major ionization channels 


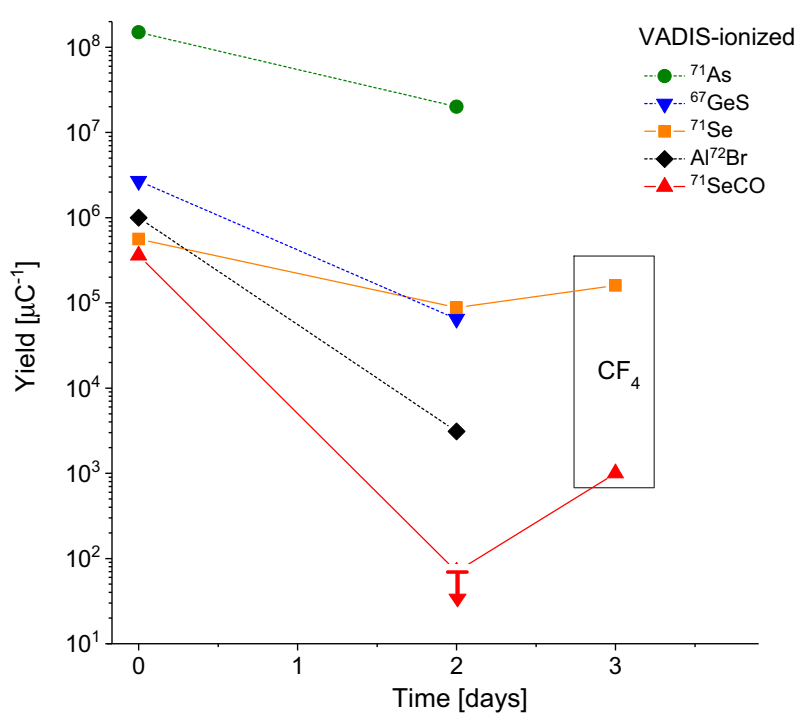

Fig. 4. Evolution of yields: After two days, the yield of ${ }^{71} \mathrm{SeCO}^{+}$decreased below the detection limit of $\approx 200$ ions $/ \mu \mathrm{C}$. Injection of $\mathrm{CF}_{4}$ (after 3 days) could partially recover the beam. The connecting lines between the data points were inserted to guide the eye.

are expected to contribute to the extracted atomic $\mathrm{Se}^{+}$ beams. Besides ionization of atomic selenium, an additional contribution is given due to dissociative ionization of selenium containing molecules. A search for selenium containing molecules other than SeCO including ${ }^{71} \mathrm{SeO}$, ${ }^{71} \mathrm{SeC},{ }^{71} \mathrm{SeF},{ }^{71} \mathrm{SeF}_{2},{ }^{71} \mathrm{SeF}_{3},{ }^{71} \mathrm{SeBr},{ }^{71} \mathrm{SeCl}$ and ${ }^{71} \mathrm{SeAl}$ remained unsuccessful. The dimer $\mathrm{Se}_{2}$ as well as SSe have not been measured, however, the compounds are not predicted to be stable by the software package HSC above $1500{ }^{\circ} \mathrm{C}$ [30]. Thus, we assume that besides ionization of atomic selenium, only dissociative ionization of carbonyl selenide significantly contributes to the extracted $\mathrm{Se}^{+}$beams. The dissociative ionization can be expressed as $\mathrm{SeCO}+\mathrm{e}^{-} \rightarrow \mathrm{Se}^{+}+\mathrm{CO}+2 \mathrm{e}^{-}$. To the best of our knowledge, partial ionization cross sections of SeCO have not yet been reported. Within the offline studies prior to online operation of target units \#605 and \#612, the ratio of stable ${ }^{\text {nat }} \mathrm{SeCO}^{+}$to ${ }^{\text {nat }} \mathrm{Se}^{+}$beams was measured. The highest ratio found was, ${ }^{\text {nat }} \mathrm{SeCO}^{+}$having twice the intensity of ${ }^{\text {nat }} \mathrm{Se}^{+}$at an Anode voltage of $170 \mathrm{~V}$. The ratio of injected neutral Se and SeCO is not known. However, from this measurement, it can be concluded, that no more than one third of the SeCO is extracted as atomic selenium ion beam. The value is in agreement with a fragmentation pattern published by Marquart et al. [31], which was measured at an electron energy of $70 \mathrm{eV}$. The neutral flow of atomic selenium $n_{\mathrm{Se}}(T)$ at temperature $T$, can be conservatively estimated from the beam currents $I$ of $\mathrm{Se}^{+}$and $\mathrm{SeCO}^{+}$as

$$
n_{\mathrm{Se}}\left(T_{2}\right)=\frac{I_{\mathrm{Se}}\left(T_{2}\right)-1 / 3 I_{\mathrm{Se}}\left(T_{2}\right)}{I_{\mathrm{Se}}\left(T_{1}\right)-1 / 3 I_{\mathrm{Se}}\left(T_{1}\right)} n_{\mathrm{Se}}\left(T_{1}\right)
$$

Yields of laser-ionized selenium scaled using eq. (1) are included in fig. 3 .
Additionally the observed contaminants for different masses of the atomic and molecular beams are given in the same fashion. A significantly higher yield (factor 35) was achieved when using electron impact $\left(\mathrm{SeCO}^{+}\right)$compared to laser $\left(\mathrm{Se}^{+}\right)$ionization. The drawback of this method, as can be concluded from the overview given in fig. 3 , is the high amount of contamination after installation of the target, which was at least one order of magnitude higher than that of the species of interest. A potential contamination of sulfur is also indicated by a peak pattern in the stable beam mass spectrum in the range from 60 to $62 \mathrm{amu}$. This is the dominant beam of a freshly prepared unit, and matches the pattern of carbonyl sulfide (SCO). However, after two days the intensity of the $\mathrm{AlBr}^{+}$and $\mathrm{GeS}^{+}$contaminants reduced by factors of 300 and 40, respectively, whilst atomic $\mathrm{Se}^{+}$is only reduced by a factor of 3.5 (cf. fig. 4). The reduction of yields with operation time of the target is a common behavior of thick target units, and often attributed to a degradation of the target material during operation at elevated temperatures [32]. The precise origin of the decreasing yields in the given target and ion source setup along within the discrepancy in the values has not been studied. A depletion of a molecular sideband can be caused by a lack of one species necessary to form the sideband. For example, outgassing of aluminum prevents formation of $\mathrm{AlBr}^{+}$. Assuming continuous release of atomic selenium from the target material, the depletion of the SeCO sideband might be caused by depletion of the precursor compounds. Additionally, competitive reactions, like formation of carbonyl sulfide instead of carbonyl selenide, might contribute to a depletion of the sideband. Nevertheless, if control over molecular formation of SeCO can be achieved, the extraction of molecular selenium might provide a relatively pure beam. The yield evolution of target unit \#605 is shown in fig. 4. Two days after installation of the target unit \#605, ${ }^{71}$ Se yields above the detection limit of 200 ions $/ \mu \mathrm{C}$ could not be measured anymore on the mass of ${ }^{71} \mathrm{SeCO}$. However, two hours after injection of $\mathrm{CF}_{4}$ at a flow rate of $2.3 \times 10^{-5} \mathrm{mbar} \mathrm{L} \mathrm{s}^{-1}$ the yield of ${ }^{71} \mathrm{SeCO}^{+}$was found to have recovered to $1.0 \times 10^{3} / \mu \mathrm{C}$. Eight hours after the beginning of $\mathrm{CF}_{4}$ injection, a yield of $1.6 \times 10^{3} / \mu \mathrm{C}$ was found. Thus, the vanishing of the ${ }^{71} \mathrm{SeCO}^{+}$sideband is not related to sintering of the target material, which is in many other cases a common mechanism for material degradation.

\section{On-line yield measurements with Ta foils as target material}

Attempts were also made to extract selenium from a previously irradiated tantalum foil target (\#565), which had no mass markers attached to it. It was equipped with a Ta surface ion source operated at $2150^{\circ} \mathrm{C}$ with the target temperature set to $2100^{\circ} \mathrm{C}$. Calculations with ABRABLA [33] suggest that the production rate for ${ }^{70} \mathrm{Se}$ is a factor of $\approx 80$ less than for $\mathrm{ZrO}_{2}$. Although, as no $\mathrm{SeO}$ formation is expected, the increased proportion of atomic 


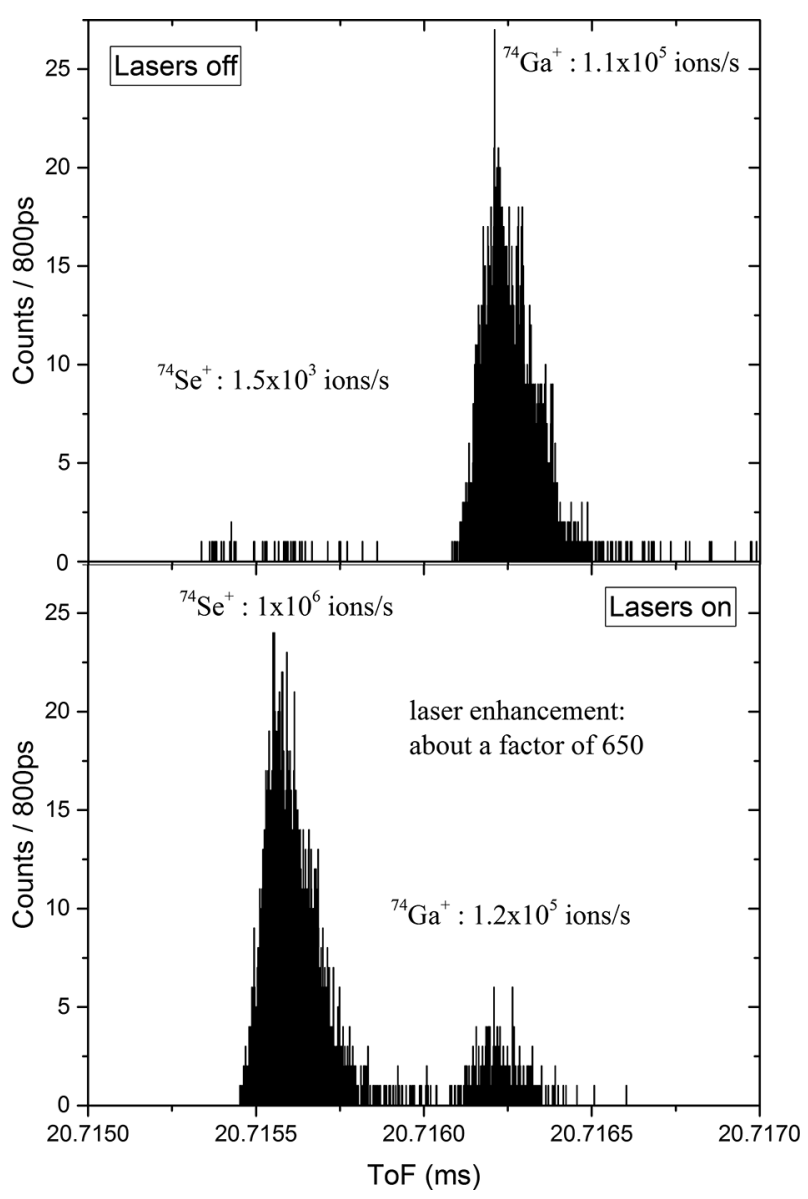

Fig. 5. $A=74$ ion beam measured with the ISOLTRAP MRToF MS without and with laser ionization using a Ta foil target (\#565). The measurements were 30 and $3 \mathrm{~ms}$ long respectively. The enhanced laser-ionized fraction of the beam (above the surface-ionized $\mathrm{Se}^{+}$and isobars) is clearly visible. The graphs were provided by F. Wienholtz.

selenium available for laser ionization combined with the standard surface ion source could lead to similar yields. With the lasers set to the according transitions (powers before launch: 1st step $80 \mathrm{~mW}$, 2nd step $2 \mathrm{~W}$, 3rd step $40 \mathrm{~W}$ ), no signal above the Faraday cup detection limit was observed for any of the stable selenium isotopes. The only signal seen was with use of the multi-reflection timeof-flight mass spectrometer (MR-ToF MS) [34], which is capable of single ion counting. With this, stable ${ }^{74} \mathrm{Se}^{+}$(radiogenic, produced in the target material) was observed, but attempts at detecting radioactive species failed. Due to the very limited amount of time for this measurement, which prohibited further optimization attempts, no conclusion about the reason for the low yield can be drawn. The high sensitivity of the MR-ToF MS allowed the measurement of both laser- and surface-ionized selenium. The enhancement factor for laser over surface ionization was 650 , and is clearly visible in fig. 5 . Also visible is the isobaric contamination composed of ${ }^{74} \mathrm{Ga}^{+}$, which was less intense than the laser-ionized selenium.

\section{Conclusion}

A RILIS ionization scheme for selenium has been tested off-line and on-line at ISOLDE, yielding an efficiency of $>1 \%$ in a hot-cavity ion source. This value is expected to reach up to $10 \%$ if a reliable means of accessing the ground state atomic population is achieved. Laser-ionized radiogenic selenium production was investigated for $\mathrm{ZrO}_{2}$ and Ta foil targets during on-line tests at ISOLDE. In the case of the $\mathrm{ZrO}_{2}$ targets the RILIS-mode of the VADLIS was compared with standard electron impact ionization of either elemental or molecular ( $\mathrm{SeCO}$ ) selenium. The ${ }^{71} \mathrm{Se}^{+}$ion beam produced by electron impact ionization was dominated by a $10 \times$ more intense background of electron-impact ionized ${ }^{71} \mathrm{As}^{+}$. Whilst in RILIS mode the production of ${ }^{71} \mathrm{Se}^{+}$was free of radioactive isobaric contamination (no detection of ${ }^{71} \mathrm{As}^{+}$), a loss of factor $\approx 35$ in the ion rate was observed compared to electron impact ionization. Taking into account non-optimal conditions of laser ionization (low population of starting level) and assuming overcoming the technical difficulty of producing stable emission of laser light at $207.5 \mathrm{~nm}$, the expected yield of laser-ionized selenium could reach the same order of magnitude as was obtained using the electron impact ionization.

For the Ta-foil target, RILIS ions were observed but the extracted ion rates were negligible, remaining below the Faraday cup detection limit. The molecular SeCO beams, extracted from the $\mathrm{ZrO}_{2}$ targets, could neither provide the required yields and purity. Additionally the observed drop of molecular formation during the period of the measurements showed that further investigations into the production of these beams need to be made.

\section{Outlook}

For a better understanding of the molecular formation, the underlying chemical processes will be investigated by thermodynamic simulations and in a dedicated experimental setup. Moreover, target materials which favor atomic Se production should be tested in combination with the standard hot-cavity RILIS configuration. Should additional time for laser ionization scheme development become available, further investigations for identifying transitions to autoionizing states can be made to improve the laser ionization scheme efficiency. Scanning the range of either the frequency-doubled dye or Ti:sapphire lasers would provide access to an unexplored energy range of the continuum.

We would like to thank F. Wienholtz and the ISOLDE ISOLTRAP team for the conduction of MR-ToF measurements, and their persistent support during the online tests. We also acknowledge support and separator setup by the ISOLDE operating staff. This project has received funding from the European Union's Horizon 2020 research and innovation program under grant agreement No. 654002. 
Data Availability Statement This manuscript has no associated data or the data will not be deposited. [Authors' comment: All data can be made available by the authors upon reasonable request.]

Publisher's Note The EPJ Publishers remain neutral with regard to jurisdictional claims in published maps and institutional affiliations.

Open Access This is an open access article distributed under the terms of the Creative Commons Attribution License (http://creativecommons.org/licenses/by/4.0), which permits unrestricted use, distribution, and reproduction in any medium, provided the original work is properly cited.

\section{References}

1. M.J.G. Borge, B. Jonson, J. Phys. G 44, 044011 (2017).

2. R. Catherall et al., J. Phys. G 44, 094002 (2017).

3. U. Köster et al., in Exotic Nuclei and Atomic Masses (ENAM 98) (ASCE, 1998) pp. 989-994.

4. L. Penescu et al., Rev. Sci. Instrum. 81, 02 A906 (2010).

5. V.I. Mishin et al., Nucl. Instrum. Methods B 73, 550 (1993).

6. D.A. Fink et al., Nucl. Instrum. Methods B 344, 83 (2015).

7. T. Day Goodacre et al., Nucl. Instrum. Methods B 376, 39 (2016)

8. Y. Martinez Palenzuela et al., Nucl. Instrum. Methods B 431, 59 (2018).

9. A. M. Hurst et al., Phys. Rev. Lett. 98, 072501 (2007).

10. D. Doherty, J. Ljungvall, Technical report CERN-INTC2014-057. INTC-P-423 (2014).

11. N. Orce et al., Technical Report CERN-INTC-2012-067. INTC-P-368 (2012).

12. U. Köster et al., Nucl. Instrum. Methods B 204, 303 (2003).
13. F. Wenander, JINST 5, C10004 (2010).

14. V.N. Fedosseev et al., J. Phys. G 44, 084006 (2017).

15. T. Day Goodacre et al., Nucl. Instrum. Methods A 830 , $510(2016)$.

16. T.E. Cocolios et al., Nucl. Instrum. Methods B 266, 4403 (2008).

17. NIST database, extracted January (2017).

18. J.E. Ruedy, R.C. Gibbs, Phys. Rev. 46, 880 (1934).

19. C. Morillon, J. Vergès, Phys. Scr. 10, 227 (1974).

20. V.N. Fedosseev et al., Opt. Spectrosc. 57, 552 (1984).

21. T. Day Goodacre et al., Nucl. Instrum. Methods A 830 , 510 (2016)

22. H.L. Ravn, S. Sundell, L. Westgaard, Nucl. Instrum. Methods 123, 131 (1975).

23. B. Marsh, Contribution to the CAS-CERN Accelerator School: Ion Sources, Senec, Slovakia, 29 May - 8 June 2012, https://cds.cern.ch/record/1445287 (CERN, 2013).

24. E. Hagebø et al., Nucl. Instrum. Methods B 70, 165 (1992).

25. S. Sundell, H. Ravn, Nucl. Instrum. Methods B 70, 160 (1992).

26. T.G. Pearson, P.L. Robinson, J. Chem. Soc. (Resumed), pp. 652-660 (1932).

27. L. Penescu, Techniques to produce and accelerate radioactive ion beams, $\mathrm{PhD}$ Thesis, Bucharest, Polytechnic Inst (2009).

28. M. Turrión et al., Nucl. Instrum. Methods B 266, 4674 (2008).

29. R Kirchner, Nucl. Instrum. Methods B 70, 186 (1992).

30. A. Roine, HSC Chemistry 7.1. http://www.hscchemistry.net (2010) (online; accessed 19 July 2015).

31. J.R. Marquart, R.L. Belford, H.A. Fraenkel, Int. J. Chem. Kinet. 9, 671 (1977).

32. J.P. Ramos, Nucl. Instrum. Methods B, https:// doi.org/10.1016/j.nimb.2019.05.045.

33. A. Kelic et al., arXiv:0906.4193 (2009).

34. R.N. Wolf et al., Nucl. Instrum. Methods A 686, 82 (2012). 